\title{
Reaction behaviour of peptide-based single thiol-thioesters exchange reaction substrate in the presence of externally added thiols
}

\author{
Makafui Y. Folikumah, Institute of Active Polymers and Berlin-Brandenburg Centre for Regenerative Therapies, Helmholtz-Zentrum Hereon, Kantstr. 55, \\ 14513 Teltow, Germany; Institute of Chemistry, University of Potsdam, 14476 Potsdam, Germany \\ Marc BehI $\mathbb{D}$, Institute of Active Polymers and Berlin-Brandenburg Centre for Regenerative Therapies, Helmholtz-Zentrum Hereon, Kantstr. 55, 14513 Teltow, \\ Germany \\ Andreas Lendlein (iD, Institute of Active Polymers and Berlin-Brandenburg Centre for Regenerative Therapies, Helmholtz-Zentrum Hereon, Kantstr. 55, \\ 14513 Teltow, Germany; Institute of Chemistry, University of Potsdam, 14476 Potsdam, Germany \\ Address all correspondence to Andreas Lendlein at andreas.lendlein@hereon.de
}

(Received 5 March 2021; accepted 13 April 2021; published online: 14 July 2021)

\section{Abstract}

Identification of patterns in chemical reaction pathways aids in the effective design of molecules for specific applications. Here, we report on model reactions with a water-soluble single thiol-thioester exchange (TTE) reaction substrate, which was designed taking in view biological and medical applications. This substrate consists of the thio-depsipeptide, Ac-Pro-Leu-Gly-SLeu-Leu-Gly-NEtSH (TDP) and does not yield foul-smelling thiol exchange products when compared with aromatic thiol containing single TTE substrates. TDP generates an $\alpha, \omega$-dithiol crosslinker in situ in a 'pseudo intramolecular' TTE. Competitive intermolecular TTE of TDP with externally added "basic" thiols increased the crosslinker concentration whilst "acidic" thiols decreased its concentration. TDP could potentially enable in situ bioconjugation and crosslinking applications.

\section{Introduction}

Dynamic covalent systems are inherently more robust than their supramolecular analogues, and their reaction dynamics, which often include an on/off switchability, are generally responsive to external stimuli such as temperature and $\mathrm{pH} .{ }^{[1,2]}$ These systems are easily reproducible, straightforward to perform, and have a broad applicability as well as tolerance towards other functional groups and reaction conditions especially with respect to solvents. The thiol-thioester exchange (TTE) is such a reaction, with rapid reaction kinetics in biochemical systems and being considered the "gold standard" of this subset of dynamic covalent linkages. ${ }^{[3]}$ In some systems, the high efficiency of the reaction is evidenced by the often stoichiometric (1: 1 thiol: thioester) interchange of functionality at low concentrations and in the presence of multiple functional groups at room temperature and in aqueous environments. ${ }^{[4-6]}$ The application of TTE in material chemistry particularly in the synthesis of polythioester-based hydrogels require the use of reaction partners in high concentrations, extremely basic catalysts and the presentation of both thioester and thiol functionalities in separate and mostly hydrophobic molecules. ${ }^{[7-9]}$ In the few examples where both functionalities were present in a single molecule, the requirement of highly specific ring sizes close to the thioester to drive the equilibrium in the desired direction, limits their use in aqueous or biological environments. Single TTE reaction substrates function by a 'pseudo intramolecular exchange' where two exact molecules react with each other. These substrates are normally designed by the incorporation of thioesters derived from more acidic thiols (lower $\mathrm{pKa}$ ) to facilitate easy exchange with thiols of higher $\mathrm{pKa}$ on the same molecule. Therefore, the exchangeable acyl group is preferentially accommodated by the less acidic thiol in TTE reactions of substrates, which exhibit a substantial difference in the acidity of thioester and attacking thiols. For this purpose, thioesters derived from aromatic thiols are commonly employed. But this presents challenges for their potential use in biological systems because of limited solubility in aqueous media, toxicity and liberation of foul-smelling thiols in the exchange reactions. ${ }^{[8]}$

Our approach towards thiol-based compounds aims at a latent crosslinker, which can form polymer networks in biological systems when exposed to a suitable stimulus. Our concept of a biologically derived and relevant peptide mimetic integrates both functionalities required for a 'pseudo' intramolecular exchange reaction in one substrate without incorporating aromatic acidic thiols.

Hence the thio-peptide mimetic shall comprise a thioester unit and a free thiol moiety with a sequence that can promote exchange reactions at neutral to slightly basic $\mathrm{pHs}$ to yield a thio-peptide mimetic with $\alpha, \omega$-free thiols, which are suitable for a crosslinking reaction with multi-arm macromolecular precursors bearing orthogonal thiol functionalities.

Designing a peptide-based TTE substrate for aqueous environments requires careful balancing of the peptide sequence structure to facilitate solubility and successful exchange of thioester acyl group. We selected the water-soluble collagenase 
substrate, Ac-Pro-Leu-Gly-SLeu-Leu-Gly-OEt ${ }^{[10]}$ with a thioleucine derived thioester unit as a reference compound. Amidation of the C-terminal end with a cysteamine unit yielded a TTE amenable thio-depsipeptide, Ac-Pro-Leu-Gly-SLeu-Leu-GlyNEtSH (TDP) bearing a free thiol with higher expected pKa than that of thioester thio-leucine.

A higher pKa value of the attacking thiolate ion than that of the displaced or departing has been noted as a requirement for the breakdown of the tetrahedral intermediate to form exchanged products. ${ }^{[11]}$ In line with $p K a$ requirements ${ }^{[12]}$ for TTE reactions and from steric considerations, the Ac-Pro-LeuGly- acyl unit should preferentially rest on the cysteamine thiol assuming a lower $\mathrm{pKa}$ value than that of the thioester derived thiol. In the TTE, a mixture of products including a rearranged thio-depsipeptide (RTDP) and $\alpha, \omega$-free thiol bearing -Leu-LeuGly- unit (BTDP) orthogonal to Michael acceptor functionalized multi-arm macromolecules should be generated.

The combination of the sensory capability of sulfhydryl chemistry towards $\mathrm{pH}$ changes and the dynamic nature of TTE reaction make single TTE substrates ideal candidates for the on-demand synthesis of active compounds. Acetylcholinesterase (AChE) in conjunction with TTE was exploited for the rapid generation and screening of dynamic library of substrates in a process, which resulted in the amplification of the best substrate. ${ }^{[6]}$

The importance of TTE reactions in biomaterial synthesis was recently demonstrated using TDP as latent, $\mathrm{pH}$-sensitive crosslinker to form a hydrogel with multi-arm polyethylene glycol maleimide in a process analogous to the activity of prodrugs. ${ }^{[13]}$ The report explained how this sequentially coupled functional system could be beneficial in the treatment of inflamed tissues associated with urinary tract like bladder infections for which $\mathrm{pH}$ levels above $7^{[14]}$ were reported. The applicability of our designed TDP is however not limited to $\mathrm{pH}$-sensitive crosslinking reactions, also a potential system for the rapid identification of thiols by dynamic combinatorial screening as shown in Scheme 1 can be thought of.

To effectively exploit the entire application spectrum of the designed TDP, knowledge of the fate of exchange products of TDP in the presence of thiols other than the TDP thiol (herein referred to as external thiols) is required. A detailed investigation on the predictability of TDP TTE reactions in the presence of external thiols would be a valuable reference point for the application of the TDP substrate in biological systems, where other (external) thiol might be part of the physiological environment.

In model reactions, we probe the exchange of TDP in the presence and absence of a set of thiols according to Scheme 1 . The presence of external thiols results in two competing reactions namely the 'pseudo' intramolecular reaction of two TDP molecules and the intermolecular reaction of a TDP molecule and an external thiol. The thiols were carefully selected to give an overview of their reactivity and ability to preferentially stabilize the exchangeable acyl unit with the regards to overall structure and $\mathrm{pKa}$ of the thiols. TDP TTE reactions with two of the most abundant thiols in biological systems, L-cysteine (LCYS), and L-glutathione (LGLU) together with a small molecular weight thiol, methylthioglycolate (MeTGC) were studied. Although these thiols have similar acidities compared to TDP, their small size relative to TDP should the outcome of the overall TTE reaction. Para-nitrothiophenol ( $p$-NTP) and 4-mercaptobenzoic acid (4MBA) were selected as acidic thiols to compare the effect of pKa of the attacking thiols on the exchange reaction.

Reaction mixtures were analysed with ESI-MS, which was run in both ion modes as it was found in preliminary measurements that the reaction products ionize preferentially only in one of the ion modes. These were complemented with ${ }^{1} \mathrm{H}$ and 2D-NMR experiments (COSY and multiplicity edited HSQC) where necessary.

\section{Materials and methods}

L-cysteine (97\%), L-glutathione (98\%), 4-mercaptobenzoic acid (99\%), methylthioglycolate (95\%) and $\mathrm{MeCN}^{-\mathrm{d}_{3}}(99.8 \%)$ and $\mathrm{D}_{2} \mathrm{O}(99.0 \%)$ were purchased from Aldrich Chemicals (Darmstadt, Germany) and were used as received. Para-nitrothiophenol (99\%) and deuterated dimethyl sulfoxide, DMSO- $\mathrm{d}_{6}$ (99.8\%) were purchased from Alfa-Aeser (Kandel, Germany) and VWR Chemicals (Darmstadt, Germany), respectively. MeOH-d ${ }_{4}(99.8 \%$ ) was purchased from Carl Roth (Karlsruhe, Germany). TDP was synthesized according to the method described in Ref. 13. In brief, the multi-step synthesis was accomplished by the initial coupling of Fmoc-Leu-Gly-OH with 2-(tritylthio)ethanamine to yield Fmoc-Leu-Gly-NEtSTrt (1). Deprotection of the Fmoc from 1 yielded the free amine $\mathrm{H}_{2} \mathrm{~N}$-Leu-Gly-NEtS-Trt (2), which was reacted with 2-(acetylsulfanyl)-4-methylpentanoic acid (acetylated thioleucine) to obtain AcSLeu-Leu-Gly-NEtS-Trt (3). Compound 3 was deacetylated to produce HSLeu-Leu-Gly-NEtS-Trt (4). Reaction of intermediate 4 with Ac-Pro-Leu-Gly-OH yielded Ac-Pro-Leu-Gly-SLeu-Leu-Gly-NEtS-Trt (5). Detritylation and high performance liquid chromatography of $\mathbf{5}$ yielded TDP as colourless fluffy material after freeze drying.

\section{Electrospray ionization mass spectrometry (ESI-MS)}

ESI-MS (direct injection) spectra were obtained on a Bruker Impact II quadrupole/time-of-flight (QqTOF) mass spectrometer (Bruker Daltonics, Bremen, Germany) equipped with an atmospheric pressure ionization source operating in the nebulizer assisted electrospray mode. ESI-MS (direct injection) spectra were obtained in positive/negative ion mode by direct injection of samples into the system using a syringe pump (Cole-Parmer, Vernon Hills, IL), which was operated at a flow rate of $180 \mu \mathrm{L} \cdot \mathrm{h}^{-1}$. Internal calibration of the system was carried out using standard sodium formate mixture.

TTE reactions of TDP were carried out using $1 \mathrm{mM}$ stock solutions of TDP and thiols in milli-Q water or ESI-grade $\mathrm{MeOH}$ where specified. $300 \mu \mathrm{L}$ of $1 \mathrm{mM}$ TDP solution and 


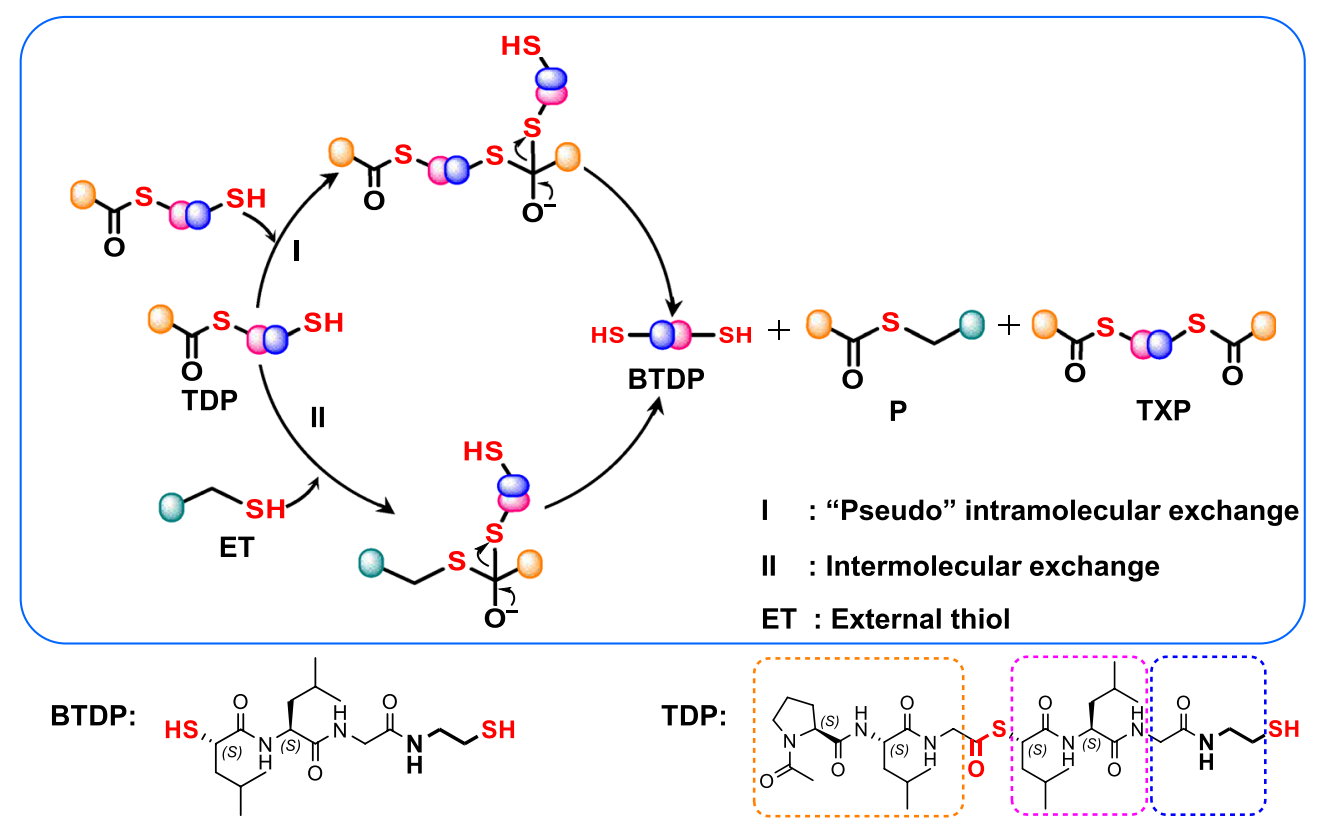

(a)

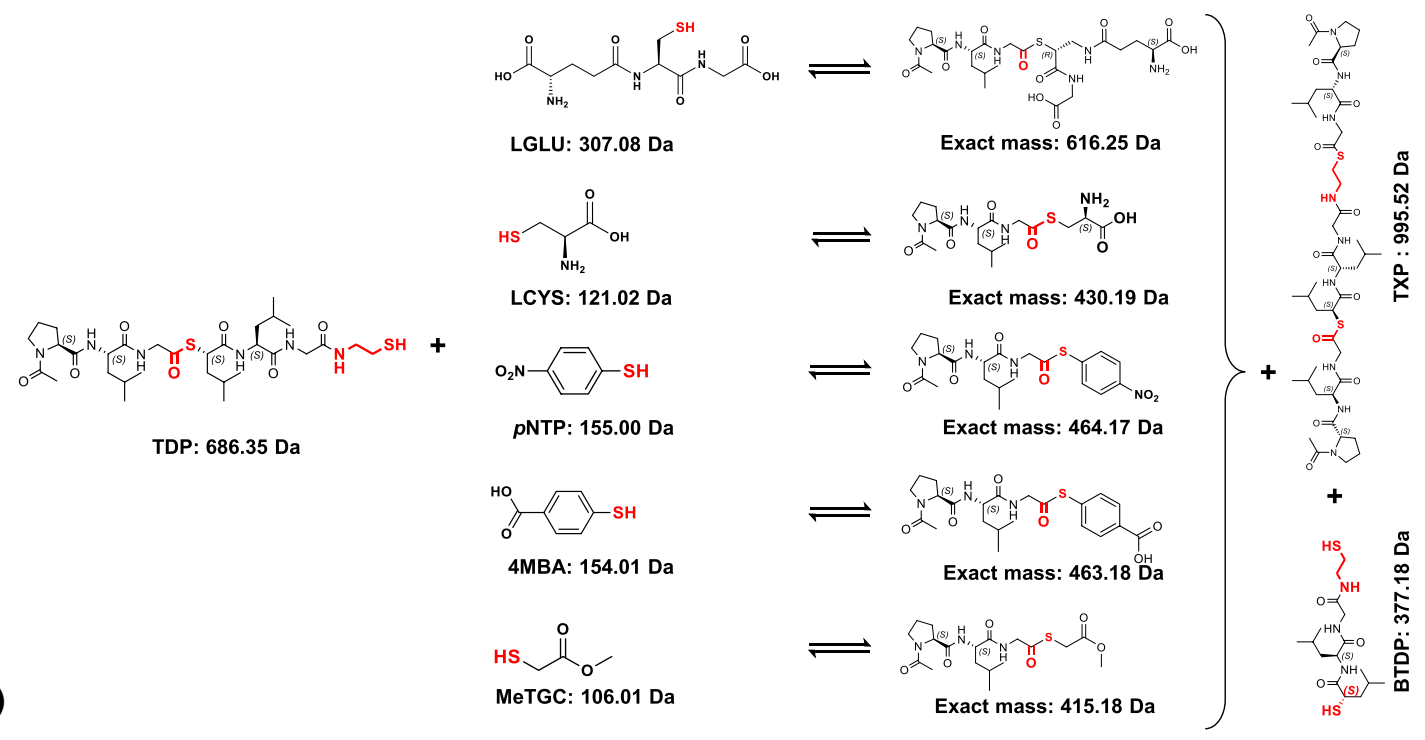

Scheme 1. (a) TDP-TDP "pseudo" intramolecular TTE reaction in the absence of external thiols (I). In the presence of external thiols, a competing TDP-ET intermolecular TTE reaction (II) as well as (I) can occur resulting in the generation of mixed exchange products BTDP, $\mathrm{P}$, and TXP. The relative concentration of exchange products depends on the relative acidities of the TDP and the external thiols. (b) TDP TTE reactions with external thiols (LGLU, LCYS, pNTP, 4MBA and MeTGC).

$300 \mu \mathrm{L}$ of $1 \mathrm{mM}$ solution of each commercial thiol were reacted for $30 \mathrm{~min}$. The reaction mixture was diluted 20 -fold to obtain a final concentration of $0.05 \mathrm{mM}$ (with respect to each reactant) before the mixture was injected into the mass spectrometer for mass analysis.

All data were processed with the software Bruker Compass Data Analysis 4.3 (Bruker Daltonics, Bremen, Germany) and Mestrenova 12.0 (Mestrelab Research, S.L., Santiago de Compostela, Spain).

\section{Nuclear magnetic resonance (NMR)} spectroscopy

${ }^{1} \mathrm{H}-\mathrm{NMR}$ (500 and $700 \mathrm{MHz}$ ) and ${ }^{13} \mathrm{C}-\mathrm{NMR}(101 \mathrm{MHz})$ were recorded in $\mathrm{D}_{2} \mathrm{O}$, in $\mathrm{MeCN}_{-} \mathrm{d}_{3}$ (internal standard: $1.94 \mathrm{ppm},{ }^{1} \mathrm{H}$; $118.3 \mathrm{ppm},{ }^{13} \mathrm{C}$ ), in DMSO-d ${ }_{6}$ (internal standard: $2.50 \mathrm{ppm},{ }^{1} \mathrm{H}$; $39.52 \mathrm{ppm},{ }^{13} \mathrm{C}$ ), in MeOD- $\mathrm{d}_{3}$ (internal standard: $3.31 \mathrm{ppm}$, ${ }^{1} \mathrm{H} ; 49.15 \mathrm{ppm},{ }^{13} \mathrm{C}$ ), on a Bruker Avance- $500 \mathrm{MHz}$ or 
Ascend-700 MHz spectrometer. Chemical shifts $(\delta)$ were reported as parts per million (ppm). Atoms are numbered with respect to all major connecting points on each molecule. The labels therefore refer to all the different atoms at each point.

\section{Results and discussion Electrospray ionization mass spectrometry}

Although the synthesized TDP was thoroughly characterized and identified with detailed NMR studies in polar aprotic solvents like $\mathrm{ACN}-\mathrm{d}_{3}$ and DMSO- $\mathrm{d}_{6}$ as previously reported, ${ }^{[13]}$ in polar protic solvents a mixture of structures were observed when NMR spectra of TDP were obtained. This observation is however expected for systems with no additional catalyst to promote the TTE reaction. To comprehensively understand the nature and fate of exchange products of TDP, additional TTE reactions in which a set of model thiols was mixed with TDP in equimolar quantities was carried out. Since the thioester unit is constant, it was speculated that by careful selection of the model thiols (pKa and structure considered), the TTE equilibrium position and kinetics should be influenced.

To a large extent, all TTE reaction of TDP yielded the expected results. TDP undergoes a "pseudo" intramolecular TTE in which the thiol of the cysteamine unit with anticipated high pKa attacks the thioester of another TDP molecule resulting in BTDP and TXP as the exchange products in the equilibrium reaction (Fig. 1).

When TDP was mixed with the selected thiols, the fate of exchange products was found to be dependent on the relative acidities of the TDP thiol and the external thiol. As an example, in the presence of L-cysteine, TDP yields an additional product peak, which is visible in the negative ion mode at $\mathrm{m} / \mathrm{z}=429.1819 \mathrm{Da}$ as an intense peak and as a weak signal at $\mathrm{m} / \mathrm{z}=453.1791 \mathrm{Da}$ in the positive ion mode (Fig. 2).

A mass peak at $\mathrm{m} / \mathrm{z}=1115.5263$ Da was initially assigned to the $\left[\mathbf{P}_{\mathbf{2 1}}+\mathbf{B T D P}\right]$ ion cluster as an evidence of a subsequent $\mathrm{S} \rightarrow \mathrm{N}$ acyl transfer of $\mathbf{P}_{\mathbf{1}}$. $\mathbf{P}_{\mathbf{2}}$ would have been formed by TTE of $\mathbf{P}_{\mathbf{2}}$, as a product of the $\mathrm{S} \rightarrow \mathrm{N}$ acyl transfer of $\mathbf{P}_{\mathbf{1}}$. Mechanistic studies on TTE reactions however suggests the presence

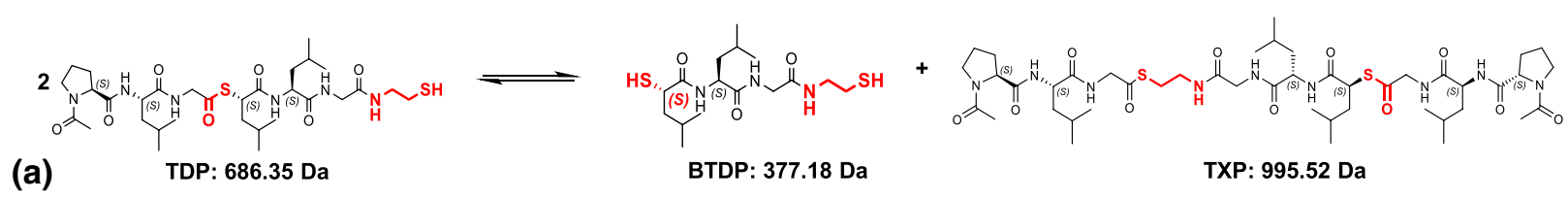

(b)

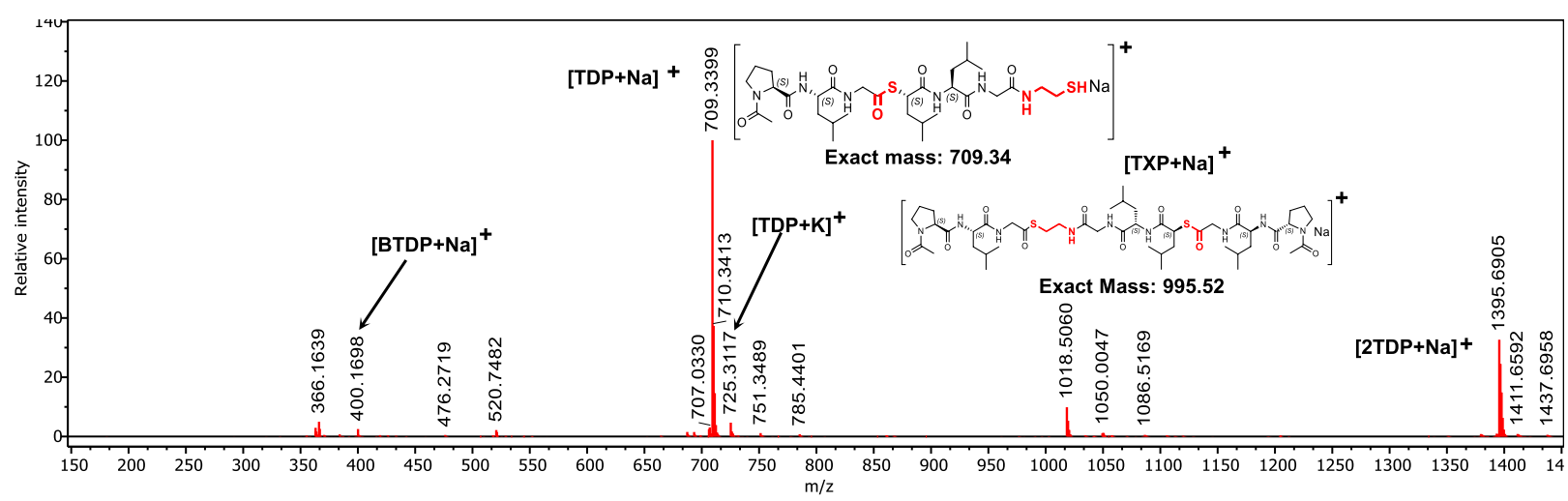

(c)

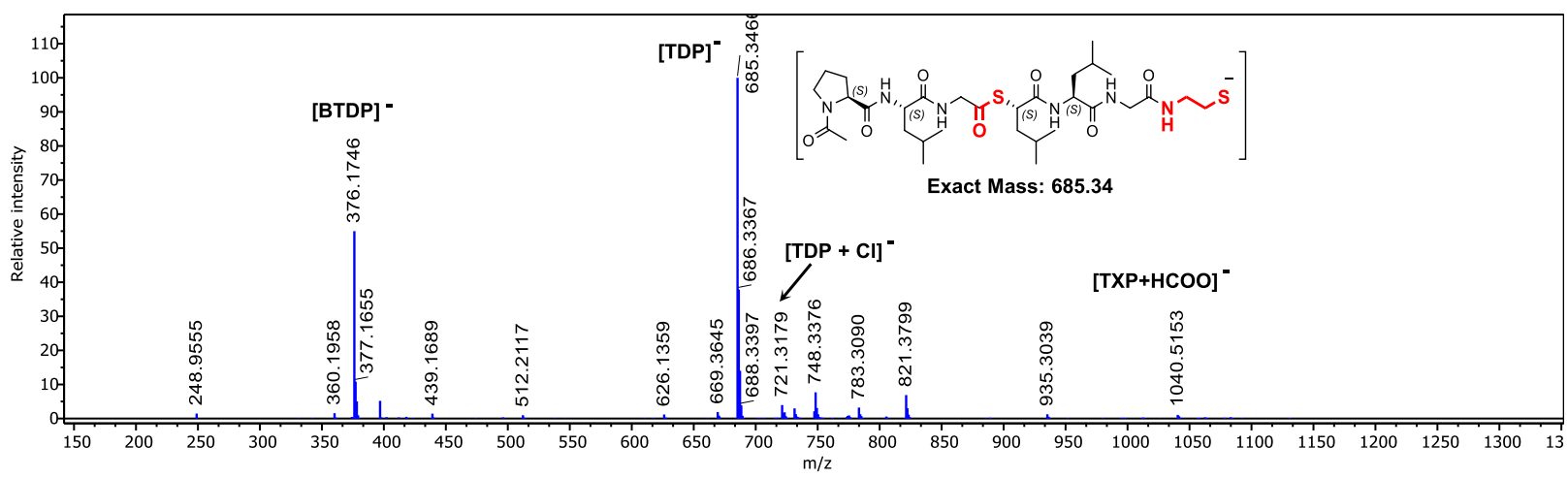

Figure 1. (a) TTE of TDP only. The scheme details TDP in equilibrium with BTDP and TXP as TTE products. Recorded mass spectra in positive ion mode (b) and negative ion mode (c) show reactant and product peaks with assignments. 


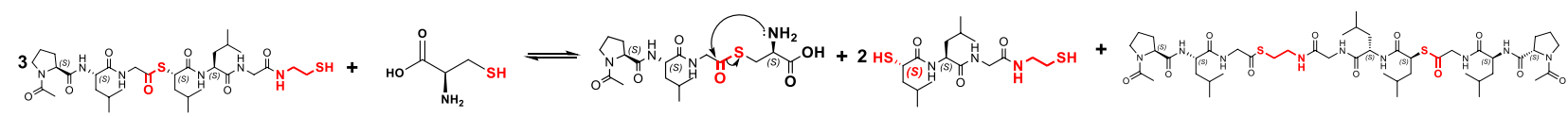
TDP: $686.35 \mathrm{Da}$ LCYS: $121.02 \mathrm{Da}$

(b)

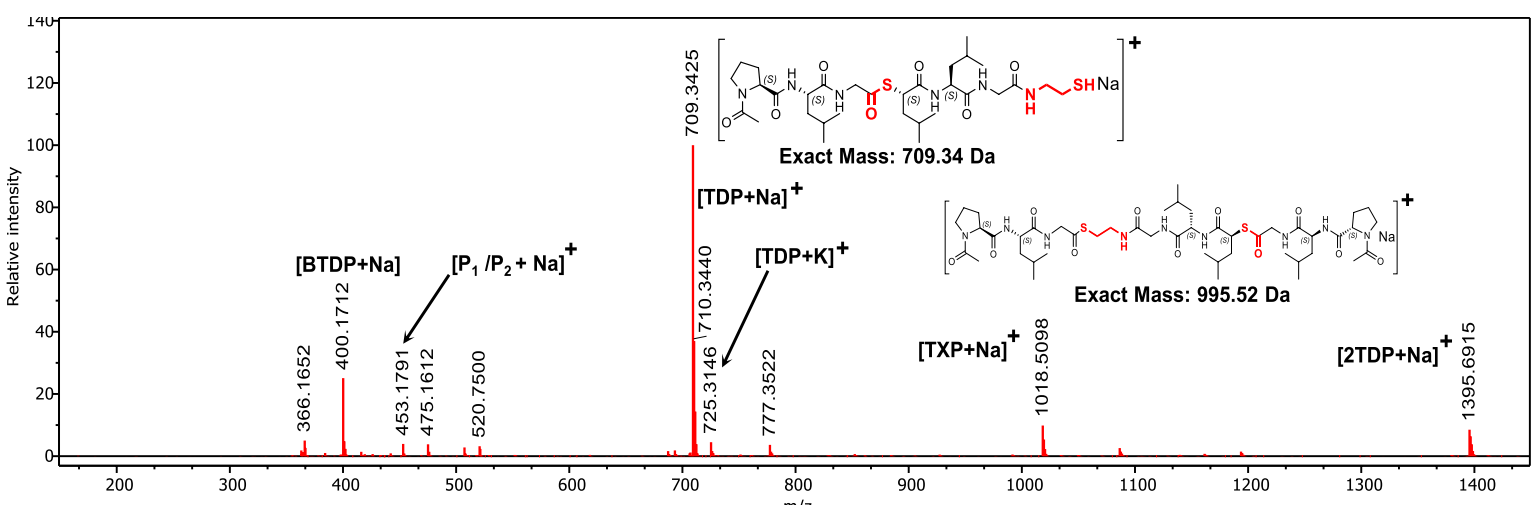

(c)

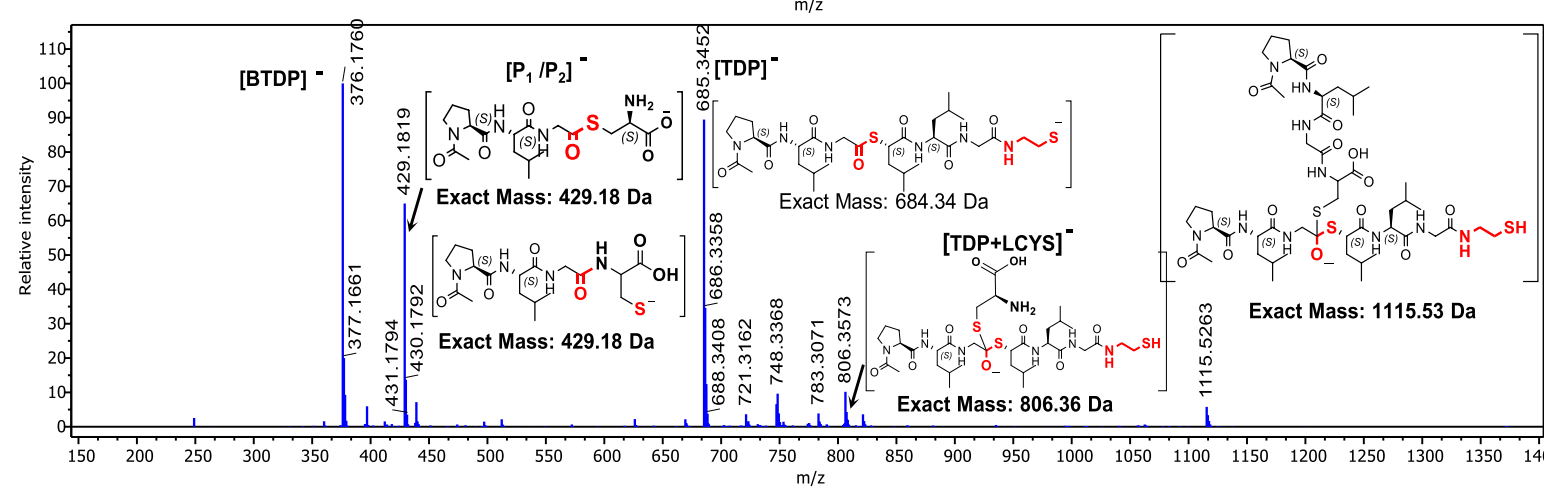

Figure 2. (a) TTE reaction of L-cysteine (LCYS) with TDP. The scheme details the reaction partners with potential $\mathrm{S} \rightarrow \mathrm{N}$ acyl transfer of the formed exchanged product. Recorded mass spectra in positive ion mode (b) and negative ion mode (c) show reactant and product peaks with assignments.

of two reaction steps leading the final exchange product with the first step being with the formation of a negatively charged tetrahedral intermediate. The mass peaks at $m / z=806.3573 \mathrm{Da}$ and 1115.5263 $\mathrm{Da}$ were therefore assigned to the tetrahedral intermediates of TDP with cysteine and the $\mathrm{S} \rightarrow \mathrm{N}$ acyl transfer product, $\mathbf{P}_{\mathbf{2 1}}$ in line with the findings from these mechanistic studies. This assumption is confirmed not only by the observed masses, but also by the fact that these ions were only observed in the negative ion mode since the intermediates are always negatively charged. The ability to detect such intermediates could be also be explained by the relative $\mathrm{pKa}$ values of the incoming and departing thiols as the important parameter determining the half-life of the intermediates.
In the presence of L-glutathione, the expected TTE product peak was not observed in both ion modes. However, a mass peak with $\mathrm{m} / \mathrm{z}=992.4252 \mathrm{Da}$ corresponding to the LGLU-TDP intermediate was present (Figure S1, Supplementary Information). The lack of a mass peak for the expected TTE product would have been accompanied by the corresponding lack of a BTDP ion peak if the only source of BTDP would be the breakdown of the LGLU-TDP tetrahedral intermediate. The presence of a relatively intense BTDP peak therefore implies the existence a competing TTE reaction by TDP molecules resulting in intermediates, which break down faster compared to LGLU-TDP. 
Unlike LCYS and LGLU, the product peak of the reaction of methylthioglycolate (MeTGC) and TDP (MeTGC-TDP) was only found in the positive ion mode at $\mathrm{m} / \mathrm{z}=438.1676 \mathrm{Da}$ (Figure S2, Supplementary Information). Signals corresponding to BTDP, TDP, and the expected TTE product, all with sodium counter ion were observed although the tetrahedral intermediate MeTGC-TDP was not found. The absence of MeTGC-TDP intermediate could be explained by a potential fast breakdown, resulting in the expected TTE products and BTDP.

The signal intensity of BTDP relative to that of TDP was found to be higher for the MeTGC-TDP reaction compared to the signals obtained when TDP was mixed with LGLU, LCYS, $4 \mathrm{MBA}$ and $p$ NTP. Since the pKa of the thiols of MeTGC, LGLU, and LCYS are comparatively similar; the observed shift towards product formation could be explained by the small size and reduced steric hindrance of the MeTGC compared to rest of the thiols.

Reaction of 4-mercaptobenzoic acid (4MBA) providing a thiol group of $\mathrm{pKa}=5.9$ was expected to form 4MBA-TDP tetrahedral intermediate with faster kinetics than LCYS, LGLU and MeTGC. The breakdown of the intermediate is slow because of the low pKa value of MBA compared to the departing thiol from TDP. The negatively charged intermediate is hence still visible at $\mathrm{m} / \mathrm{z}=839.3223 \mathrm{Da}$ (Figure S3, Supplementary Information).

The intensity of BTDP ions compared to those observed in the reaction of LGLU and TDP is low. Similarly, as it was observed for the LGLU-TDP reaction, the competing TDP-TDP TTE reaction resulted in BTDP mass peak with lower intensity than observed for the LGLU-TDP reaction. This is due to the low $\mathrm{pKa}$ value of 4MBA compared to the $\mathrm{pKa}$ of TDP, which significantly reduces the likelihood of TDP-TDP intermediate formation since the 4MBA-TDP would be preferentially formed. Like 4MBA, para-nitrothiophenol ( $p$ NTP) with thiol $\mathrm{pKa}$ of 4.6 also yielded a mass peak corresponding to the $p$ NTP-TDP intermediate at $\mathrm{m} / \mathrm{z}=840.3467 \mathrm{Da}$ only in the negative ion mode (Figure S4, Supplementary Information).

\section{Nuclear magnetic resonance spectroscopy}

ESI-MS is known to operate via non-destructive ionization of analytes being studied and is a more sensitive analytical technique compared to NMR. There is however some loss of information due to the fact that analytes are in direct interaction with the instrument leading to certain undesired modifications. For simple to moderately complicated systems, NMR serves an additional or complementary tool to obtain valuable information of analytes in their pristine condition. ${ }^{1} \mathrm{H}-\mathrm{NMR}$ was therefore used to study the TDP TTE reaction mixtures in similar experiments as performed for ESI-MS study. For each thiol, NMR spectra were collected for pristine thiol, TDP only solution and for the TDP- external thiol mixture in deuterium oxide or methanol- $\mathrm{d}_{4}$ depending on the solubility of the selected thiol. Methanol- $\mathrm{d}_{4}$ was used for reactions involving $4 \mathrm{MBA}$ and $p \mathrm{NTP}$ due to their limited solubility in $\mathrm{D}_{2} \mathrm{O}$.
For each solvent, the corresponding TDP only spectrum was obtained to enable a true comparison. Spectra for TDP only solution were taken after $30 \mathrm{~min}$ of standing whilst spectra for TDP-external mixtures were taken after 30 min of mixing and repeated after $12 \mathrm{~h}$. Resonances for methylene protons on the cysteamine unit of the TDP and BTDP are labelled TD $_{1}$ and $\mathbf{T D}_{\mathbf{2}}$ whilst the peaks denoted with $\mathbf{T} \mathbf{X}_{\mathbf{1}}$ and $\mathbf{T} \mathbf{X}_{\mathbf{2}}$ are resonances associated with the methylene protons on the cysteamine unit TXP (Fig. 3a).

Figure 3(b) presents an overlay of ${ }^{1} \mathrm{H}-\mathrm{NMR}$ spectra of LCYS, TDP only (after $30 \mathrm{~min}$ ), TDP-LCYS (after $30 \mathrm{~min}$ ) and TDP-LCYS (after $12 \mathrm{~h}$ ) in $\mathrm{D}_{2} \mathrm{O}$. The general trend observed in this series of spectra compare favourably with observations from the ESI-MS studies. In this regard, the acidity of the added thiol determines if TDP-TDP exchange products are formed preferentially over TDP-external thiol products or vice versa. As an example, NMR spectra TDP only solution shows the occurrence of $\mathbf{T X} \mathbf{X}_{2}$ resonance downfield of $\mathbf{T} \mathbf{D}_{2}$ and overlapping signals of $\mathbf{T} \mathbf{X}_{\mathbf{1}}$ and $\mathbf{T D}_{\mathbf{1}}$ at $\delta=3.26-3.39 \mathrm{ppm}$.

The newly formed and observed $\mathbf{T} \mathbf{X}_{2}$ signal at higher ppm value compared to $\mathbf{T D}_{\mathbf{2}}$ is an indication of a successful TDP-TDP exchange reaction to form TXP. Since the electronic environments of $\mathbf{T D}_{\mathbf{1}}$ and $\mathbf{T} \mathbf{X}_{\mathbf{1}}$ remain relatively unchanged, these signals occurred at the same ppm value as a multiplet.

In the presence of LCYS however, the exchangeable acyl group preferentially rests with cysteine leading to the absence of $\mathbf{T X}_{\mathbf{2}}$ and concomitant emergence of new cysteine $\beta$-proton resonance, $\mathbf{P}$ at $\delta=2.86 \mathrm{ppm}$. The loss of the broad $\mathbf{T D}_{\mathbf{1}} / \mathbf{T X}_{\mathbf{1}}$ overlapping peak seen for the TDP only spectrum was accompanied by the presence of an intense $\mathbf{T D}_{1}$ signal contributed largely by BTDP from LCYS-TDP reaction. This result thus signifies that TDP-LCYS TTE was more favourable than that of TDP-TDP. Based on the assumption that cysteine and TDP thiols do have comparable $\mathrm{pKa}$ values, the observed preference of TDP for LCYS could be attributed to the smaller size and hence less steric hindrance of the cysteine molecule compared to TDP. Similar size effect on the fate of the exchange product was observed for TDP-MeTGC (Figure S2, Supplementary Information) although MeTGC has a more acidic thiol (pKa 7.8) compared to cysteine ( $\mathrm{pKa} 8.3$ ).

Due to the limited solubility of the more acidic thiols, 4MBA and $p$ NTP in $\mathrm{D}_{2} \mathrm{O}$, methanol- $\mathrm{d}_{4}$ was used as solvent. The effect of solvent on the TDP-TDP reaction was directly evident in the TTE kinetics when the ${ }^{1} \mathrm{H}-\mathrm{NMR}$ spectra of TDP in $\mathrm{D}_{2} \mathrm{O}$ and methanol- $\mathrm{d}_{4}$ after $30 \mathrm{~min}$ of solution preparation are compared (Figure S6A, Supplementary Information). The $\mathbf{T X}_{\mathbf{2}}$ resonance from TXP was observed in $\mathrm{D}_{2} \mathrm{O}$ after 30 min of solution preparation but after $12 \mathrm{~h}$ in methanol- $\mathrm{d}_{4}$ although this could be faster. In polar aprotic solvents such as acetonitrile, TDP required the addition of an organic base, diisopropylethylamine to effect the exchange reaction (Figure S6B, Supplementary Information).

This observation should be largely anticipated considering the polarity of the two solvents and in line with reported findings ${ }^{[12]}$ assuming thiolate ions are the main reactive species as would be in a base catalysed reaction. 

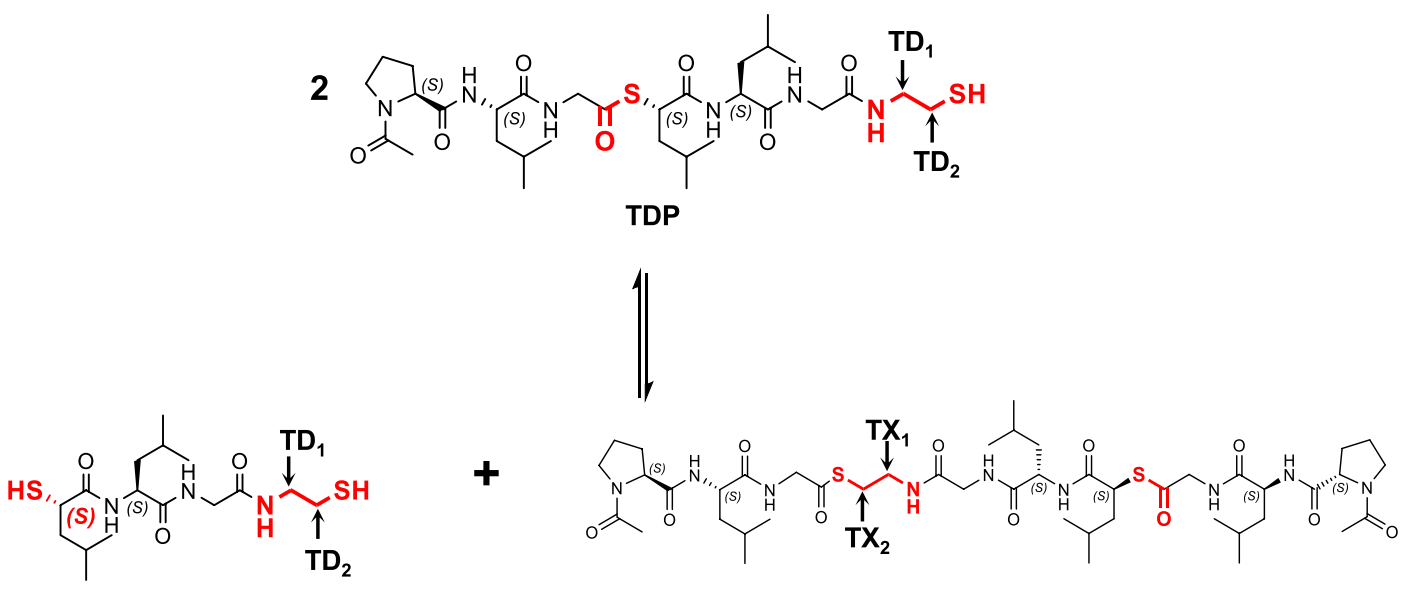

(a)

BTDP

TXP

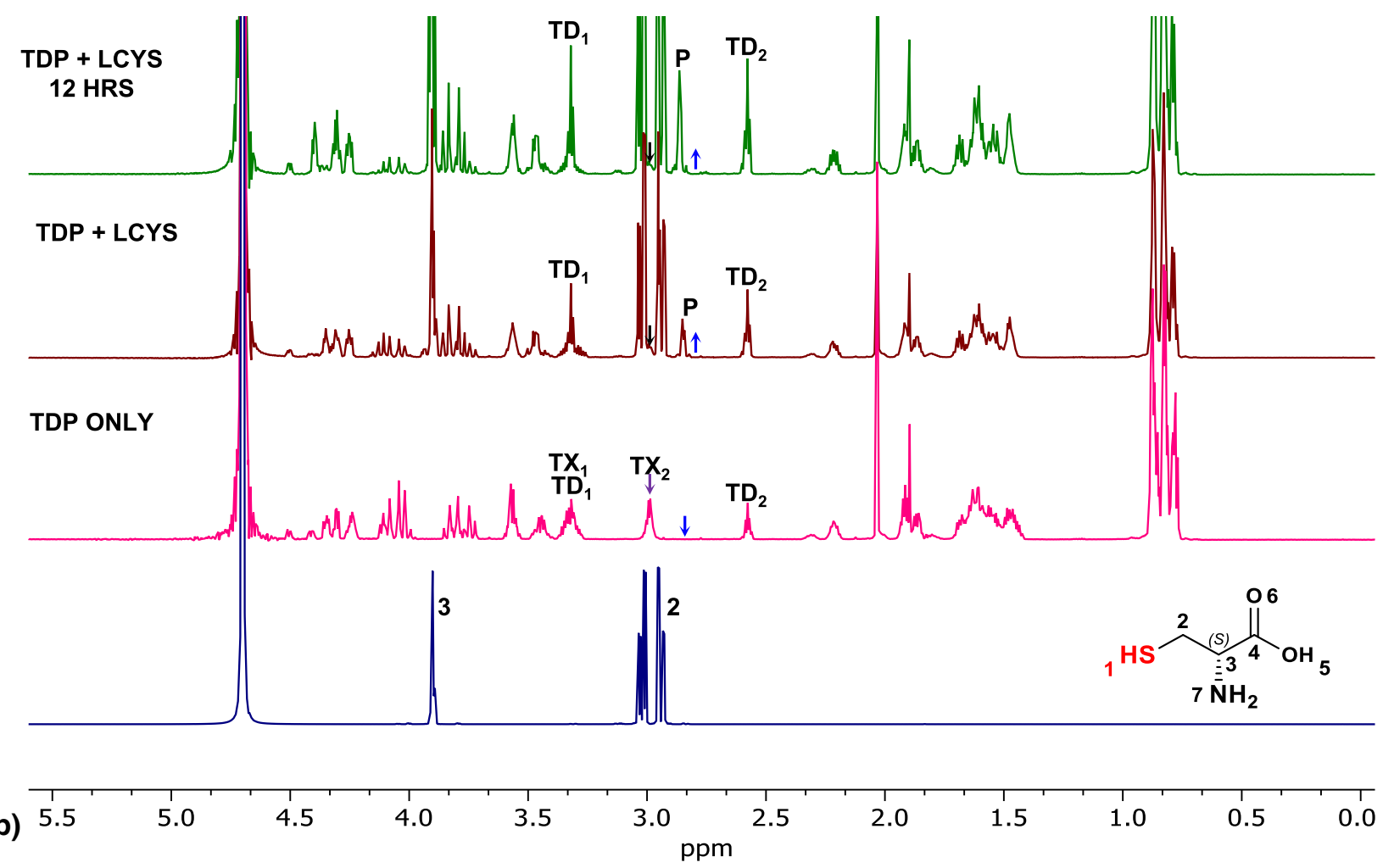

Figure 3. (a) TDP-TDP reaction scheme and labelling of relevant protons. Cysteamine protons $\mathrm{TD}_{1}$ and $\mathrm{TD}_{2}$ are assumed to have similar resonances for TDP and BTDP. (b) Overlay of NMR spectra from bottom of LCYS, TDP only (after $30 \mathrm{~min}$ ), TDP-LCYS (after 30 min) and TDP-LCYS (after $12 \mathrm{~h}$ ) in $\mathrm{D}_{2} \mathrm{O}$.

When TDP was mixed with L-glutathione, $\mathbf{T} \mathbf{X}_{2}$ resonance was not observed after $30 \mathrm{~min}$ of solution although a weak signal was present after $12 \mathrm{~h}$ (Figure S7, Supplementary Information). As was previously noted in ESI-MS studies TDP-LGLU reaction was assumed to be arrested at the initial reaction step due to the presence of a relatively intensive peak identified as the tetrahedral intermediate. If the highly intense BTDP mass peak observed for TDP-LGLU reaction was largely contributed by a competing TDP-TDP reaction, then a corresponding strong $\mathbf{T X}_{\mathbf{2}}$ resonance should be observed in the ${ }^{1} \mathrm{H}-\mathrm{NMR}$ spectrum. Since thiolate ions play significant role in TTE, these opposing findings could be attributed to the increased acid effect of the two glutathione carboxylic acid groups 
considering the final LGLU concentration used for NMR and ESI-MS studies.

Results of TTE of TDP with the more acidic thiol 4MBA (pKa 5.9) compare favourably with ESI-MS studies. In summary, due to the slow breakdown of their tetrahedral intermediates, the increased build-up of these negatively charged species could lead to the generation of TDP thiolate ions resulting in a competing TDP-TDP reaction and the presence of $\mathbf{T} \mathbf{X}_{\mathbf{2}}$ signal after 30 min (Figure S8, Supplementary Information). Similar results were obtained for $p$ NTP with a $\mathrm{pKa}$ value of 4.6 (Figure S9, Supplementary Information).

\section{Conclusions}

The thio-depsipeptide Ac-Pro-Leu-Gly-SLeu-Leu-Gly$\mathrm{NEtSH}$ (TDP) is capable to undergo a $\mathrm{pH}$ sensitive pseudo intramolecular exchange reaction producing $\alpha, \omega$-dithiol, which can then serve as a latent crosslinker or precursor. Since the thio-depsipeptide could be a candidate compound to be used for potential application in biological systems where thiol molecules are already present, an in-depth knowledge of its reactivity in the presence of some biologically relevant and organic thiols should provide valuable information for the future design of these peptide mimetics. In model TTE reactions, the reactions of TDP with L-cysteine and L-glutathione, which are two of the most abundant thiols in biological systems, methylthioglycolate, para-nitrothiophenol and 4-mercaptobenzoic acid were analysed with ESI-MS and ${ }^{1} \mathrm{H}-\mathrm{NMR}$. The model reactions of TDP with this set of external thiols confirmed the ability of TDP to undergo TTE reactions with results reflecting the role of $\mathrm{pKa}$ of thiols in the fate of exchange products. Small-sized aliphatic thiols, MeTGC and LCYS (pKas 7.8 and 8.3, respectively) promoted increased production of useful $\alpha, \omega$ - dithiol (BTDP) whilst aromatic and acidic thiols, 4MBA and $p$ NTP (pKas 5.9 and 4.6, respectively) hindered BTDP production.

Thiol-thioester exchange remains a significant reaction to several biological processes and a widely used dynamic covalent chemistry in material chemistry. Whereas the designed TDP finds potential use in bioconjugation and crosslinking reactions in biological systems, the exchange reaction results present those working in the field insight into the development of new sequences with tuneable exchange kinetics and equilibria not only for material synthesis but also in the areas of peptide mimetic probes for thiol identification and sequestration.

\section{Acknowledgments}

This work was financially supported by the Helmholtz Association through programme-oriented funding and through Helmholtz Graduate School of Macromolecular Bioscience (MacroBio, VH-GS-503).

\section{Funding}

Open Access funding enabled and organized by Projekt DEAL.

\section{Data availability}

Data will be made available on reasonable request.

\section{Declarations}

\section{Conflict of interest}

The authors declare no conflict of interest.

\section{Open Access}

This article is licensed under a Creative Commons Attribution 4.0 International License, which permits use, sharing, adaptation, distribution and reproduction in any medium or format, as long as you give appropriate credit to the original author(s) and the source, provide a link to the Creative Commons licence, and indicate if changes were made. The images or other third party material in this article are included in the article's Creative Commons licence, unless indicated otherwise in a credit line to the material. If material is not included in the article's Creative Commons licence and your intended use is not permitted by statutory regulation or exceeds the permitted use, you will need to obtain permission directly from the copyright holder. To view a copy of this licence, visit http://creativecommons. org/licenses/by/4.0/.

\section{Supplementary Information}

The online version contains supplementary material available at https://doi.org/10.1557/s43579-021-00041-z.

\section{References}

1. S.J. Rowan, S.J. Cantrill, G.R.L. Cousins, J.K.M. Sanders, J.F. Stoddart, Dynamic covalent chemistry. Angew. Chem. Int. Ed. 41, 898 (2002)

2. T. Maeda, H. Otsuka, A. Takahara, Dynamic covalent polymers: reorganizable polymers with dynamic covalent bonds. Prog. Polym. Sci. 34, 581 (2009)

3. T.M. Hackeng, J.H. Griffin, P.E. Dawson, Protein synthesis by native chemical ligation: expanded scope by using straightforward methodology. Proc. Natl. Acad. Sci. 96, 10068 (1999)

4. P.E. Dawson, T.W. Muir, I. Clark-Lewis, S.B. Kent, Synthesis of proteins by native chemical ligation. Science 266, 776 (1994)

5. M.G. Woll, S.H. Gellman, Backbone thioester exchange: a new approach to evaluating higher order structural stability in polypeptides. J. Am. Chem. Soc. 126, 11172 (2004)

6. R. Larsson, Z. Pei, 0. Ramström, Catalytic self-screening of cholinesterase substrates from a dynamic combinatorial thioester library. Angew. Chem. Int. Ed. 43, 3716 (2004)

7. T.J. Bannin, M.K. Kiesewetter, Poly(thioester) by organocatalytic ring-opening polymerization. Macromolecules 48, 5481 (2015)

8. C. Wang, S. Mavila, B.T. Worrell, W. Xi, T.M. Goldman, C.N. Bowman, Productive exchange of thiols and thioesters to form dynamic polythioester-based polymers. ACS Macro Lett. 7, 1312 (2018)

9. D. Konetski, S. Mavila, C. Wang, B. Worrell, C.N. Bowman, Production of dynamic lipid bilayers using the reversible thiol-thioester exchange reaction. Chem. Commun. 54, 8108 (2018) 
10. H. Weingarten, R. Martin, J. Feder, Synthetic substrates of vertebrate collagenase. Biochemistry 24, 6730 (1985)

11. P.J. Bracher, P.W. Snyder, B.R. Bohall, G.M. Whitesides, The relative rates of thiolthioester exchange and hydrolysis for alkyl and aryl thioalkanoates in water. Orig. Life Evol. biosph.: J. Int. Soc. Study Orig. Life 41, 399 (2011)

12. B.T. Worrell, S. Mavila, C. Wang, T.M. Kontour, C.-H. Lim, M.K. McBride, C.B. Musgrave, R. Shoemaker, C.N. Bowman, A user's guide to the thiol-thioester exchange in organic media: scope, limitations, and applications in material science. Polym. Chem. 9, 4523 (2018)

13. M.Y. Folikumah, M. Behl, A. Lendlein, Thiol-thioester exchange reaction in precursor enables $\mathrm{pH}$ triggered hydrogel formation. Biomacromolecules, 22(5), 1875-1884 (2021)

14. A. Ronald, The etiology of urinary tract infection: traditional and emerging pathogens. Dis. Mon. 49, 71 (2003) 